\title{
Variations
}

Variations

Revue internationale de théorie critique

$17 \mid 2012$

Critique du travail

\section{Critique - Brève esquisse d'une longue histoire}

\section{Michal Kozlowski}

\section{(2) OpenEdition \\ Journals}

Édition électronique

URL : http://journals.openedition.org/variations/412

DOI : 10.4000/variations.412

ISSN : 1968-3960

\section{Éditeur}

Les amis de Variations

\section{Référence électronique}

Michal Kozlowski, «Critique - Brève esquisse d'une longue histoire », Variations [En ligne], 17 | 2012, mis en ligne le 15 octobre 2012, consulté le 01 mai 2019. URL : http://journals.openedition.org/ variations/412; DOI : 10.4000/variations.412

Ce document a été généré automatiquement le 1 mai 2019.

Les ami•e•s de Variations 


\title{
Critique - Brève esquisse d'une longue histoire
}

\author{
Michal Kozlowski
}

\section{NOTE DE L'AUTEUR}

Cet article s'inspire d'une intervention faite lors d'un colloque consacré à la critique sociale selon Boltanski et Honneth à la Maison des sciences de l'homme de Sofia (Bulgarie) en 2010.

1 L'idée de fonder la critique comme un type particulier de réflexion et de connaissance sembla pendant longtemps inséparable du projet de l'émancipation. Certes, cette idée est, de manière générale, introduite par Kant mais c'est avec Marx qu'elle trouve véritablement sa place dans le champ du social et du politique. Le statut de la «critique de l'économie politique» est en lui-même une chose tout à la fois fascinante et problématique dans divers marxismes, même si Marx l'a lui-même peu théorisé. Max Horkheimer est peut-être celui qui a tenté, de manière la plus rigoureuse, de distinguer entre théorie traditionnelle et théorie critique ${ }^{1}$. Peut-on aujourd'hui conserver cette distinction et à quelles conditions? La critique est une chose que les gens font constamment - et l'on peut donc chercher avec plus ou moins de succès à théoriser cette critique, mais la théorie critique est probablement quelque chose d'assez différent. Cette théorie critique a notamment toujours cherché à se prémunir d'une manière définitive contre les renversements stratégiques opérés par les pouvoirs et les systèmes dominants. Or il semble aujourd'hui que la critique soit en fait devenue un élément essentiel de la reproduction et du renouvellement des pouvoirs et des systèmes...

2 1. La critique sociale accompagne la philosophie depuis sa naissance. Socrate lui-même en fournirait presque un idéal-type. Il représente un discours à la fois public, engagé, intransigeant et finalement tenace jusqu'à l'héroïsme. Mais dans ce champ critique qui se constitue alors comme élément sociétal distinct, la figure de Socrate annonce aussi un premier clivage qui se produit entre ses disciples directs. D'un coté Platon et sa 
République - utopie et critique englobante de la société existante, critique s'appuyant sur la connaissance suprême de la transcendance, critique morale et normative, développée par un savant qui se place à l'extérieur mais aussi au-dessus du social. De l'autre Diogène et les Cyniques, avec leur transgression des valeurs établies, la vérité non-dissimulée et attestée publiquement dans une forme violente et scandaleuse, leur message matérialiste, égalitariste et cosmopolite, prononcé depuis des positions de marginalité sociale; les cyniques ne se situant ni à l'extérieur ni vraiment à l'intérieur de cette société, mais plutôt au-dessous².

3 Il ne faudrait pas trop hâtivement associer Platon à la préfiguration d'une critique savante, voire experte, opposée à une critique populaire et inculte. L'opposition en question n'a sans doute ni prolongement, ni équivalent à notre époque. Mais elle n'en reste pas moins, pour nous, importante. Il ne s'agit pas ici de distinguer les idéaux et les valeurs affirmés, ni même d'établir une différence proprement philosophique concernant l'ontologie du social. La différence a avant tout un sens pragmatique: elle touche la manière de parler, le lieu d'où l'on parle, au nom de qui ou au nom de quoi on prend la parole... Autrement dit, le clivage qui apparait tient davantage au rapport à la vérité plus qu'à la vérité elle-même. Il tient aux modes de subjectivation que la critique produit et, finalement, aux jeux de pouvoir qui sont impliqués dans l'affaire critique. Un des problèmes, parmi beaucoup d'autres plus subtiles et nuancés, qui naît de ce clivage généalogique, touche au rapport, aussi subjectif qu'objectif, que la critique entretient avec le pouvoir - ses institutions, ses logiques internes, ses façons de légitimer les discours. Platon, comme nous le raconte la légende, a tenté de faire entrer sa critique dans la logique du pouvoir en devenant conseiller du tyran de Syracuse. Et il a gravement échoué... Les Cyniques n'ont, quant-à-eux, jamais forgé le moindre programme qui mériterait la qualification de politique. Comme si le témoignage suffisait pour accomplir la tâche critique... Ce que Luc Boltanski appelle la contradiction herméneutique de la critique $^{3}$ se trouve encore aggravée par toutes les contradictions pragmatiques et performatives de la critique. Il nous faut donc (nous qui cherchons toujours la critique) reconnaître la spécificité de cette contradiction dans notre actualité et la manière dont nous procédons pour la rendre réflexive, l'assumer et aller plus loin.

4 2. L'autre problème qui surgit de la critique dans la théorie est d'ordre épistémologique. Dans la représentation narcissique qu'elle se fait d'elle-même, la philosophie se considère comme une activité critique par excellence. Si l'essence de la philosophie réside dans l'argumentation, on peut supposer en effet que la mise à l'épreuve de la proposition et son évaluation critique mènent à l'exercice libre de la raison au service de la vérité. L'enjeu serait donc la vérité et uniquement la vérité - peu importe la manière dont on la concevrait. La critique consisterait alors à démontrer et dénoncer la non-conformité du réel (moral et sociopolitique) à cette vérité. Les grandes critiques sociales des Lumières, y compris celles de Rousseau et de Fourier, ne sortent pas de ce cadre, quand Kant révolutionne les choses en déplaçant la perspective au moins de deux manières importantes. Tout d'abord la philosophie ne doit plus selon lui s'orienter vers la recherche de la vérité mais seulement déterminer les conditions de possibilité de l'avènement de cette vérité. Par ailleurs, cette philosophie établit une rationalité distincte et propre au domaine de la normativité se détachant du diktat de la vérité. Certes la distinction entre les faits et les valeurs s'opère avant Kant (chez Spinoza et Hume en particulier) mais ce qui est décisif chez Kant, c'est précisément l'introduction de la raison 
pratique avec ses propres règles de rationalité. Ces deux concepts kantiens seront cruciaux pour la formation de la théorie critique.

En introduisant l'idée de critique de l'économie politique, Marx puise profondément dans ces deux concepts sans pour autant rendre univoque le statut épistémologique de son projet. La critique de Marx cherche à élucider les conditions de possibilité, cette fois-ci non transcendantales mais historiques et sociales, de l'économie capitaliste. Si dans un sens on peut considérer qu'il ne fait rien d'autre qu'approfondir la connaissance du capital, Marx ne prétend pas pour autant que l'économie politique du capitalisme soit tout simplement fausse. Ce qu'elle comporte d'idéologie, ce ne sont pas ses règles et ses structures, mais plus précisément ce qui la rend possible aussi bien historiquement (l'accumulation primitive) que dans son présent (l'extraction de la plus-value). En conséquence de quoi on peut dire que l'idéologie capitaliste ne ment pas, mais qu'elle refoule. Néanmoins, le vrai enjeu de la critique réside ailleurs : elle ne doit pas seulement dire la vérité, elle doit chercher les chemins de fuite - les points faibles - les possibilités de dépassement du système capitaliste. Les conditions de possibilité du capitalisme sont censées se changer en forces réelles de l'histoire, abolir le capitalisme et mener la société dans une direction déterminée, direction qui ne manque pas d'un certain caractère normatif, ce que Marx semble avoir du mal à avouer par ailleurs.

L'idée kantienne a également permis une transformation essentielle, que l'on pourrait qualifier de tournant antipositiviste, définissant un domaine autonome de Geisteswissenschaften. Celles-ci avaient pour but de découvrir les règles, non seulement spécifiques mais aussi radicalement différentes, qui libéreraient la société humaine des paradigmes mécanistes et surtout des logiques de rapport de forces dominant la compréhension positiviste de la nature. Ces nouvelles règles exigeaient une nouvelle connaissance, c'est-à-dire une nouvelle épistémologie. Elles avaient pour conséquence de produire une toute nouvelle conception de la vérité et donc de nouvelles perspectives pour la critique. Les diverses formes du transcendantalisme rendant possible un tel tournant avaient très certainement le grand mérite de s'opposer au «biologisme » et à «l'économisme» qui tentaient, et tentent toujours, de coloniser voire d'absorber les sciences sociales. Mais elles souffraient d'un dualisme héréditaire difficilement tenable. Or il me semble que nous observons aujourd'hui des tentatives progressives de reformulation du vieux postulat consistant à fonder une rationalité distincte émancipatrice, et, ensuite, à passer à des analyses plus empiriques visant à comprendre davantage les revendications critiques effectivement en marche dans le corps social.

On peut en conséquence apercevoir deux lignes différentes d'héritiers de Karl Marx. La première est formée de ceux qui ont essayé de concevoir la critique comme un type de connaissance distinct, et en quelque sorte supérieur, de la théorie simplement descriptive; au sens où cette critique est orientée vers ce qui est névralgique et ouverte à l'avenir. On retrouve sur cette ligne les noms d'Althusser, Wallerstein et Negri, signe clair qu'il ne s'agit pas ici d'une conformité politique mais théorique. La seconde ligne comprend ceux de Horkheimer, Habermas ou encore Honneth, qui se retrouvent dans une critique qui n'est jamais une simple analyse et exposition des relations de forces (même si celles-ci se dissimulent effectivement derrière une idéologie dominante). Celle-ci implique en effet toujours une revendication normative, non pas arbitraire mais fondée sur une rationalité que l'on peut qualifier de transcendantale (même si ce transcendantal est très nuancé et plus souple que chez Kant) - rationalité de l'émancipation, de la communication ou encore de la reconnaissance. Il est important de noter que dans des 
œuvres comme Strukturwandel der Öffentlichkeit ${ }^{4}$ ou Kampf um Anerkennung : Zur moralischen Grammatik sozialer Konflikte $e^{5}$, l'enjeu consiste clairement à démontrer comment l'émancipation, la communication et la reconnaissance interviennent comme des forces historiques et sociales majeures et comment la critique devient leur indispensable moyen d'expression. Certes, aucune fatalité ne garantit plus la marche historique triomphale des idées portées par l'entreprise critique, mais en tout cas elles ne sont pas moins "réelles » que les forces de domination auxquelles elles s'opposent. Cependant, la critique qui se dessine chez ses auteurs est autre chose que la résistance, le refus ou même la problématisation si chère à Michel Foucault. Elle vise les idées positives de justice, d'égalité et de vie individuelle et collective plus satisfaisantes, ce qui n'est sans doute pas sans mérite dans un monde où ces idées reculent de manière générale. Le courant critique, qui se veut immergé dans l'immanence de l'historie, plus proche du Marx tardif du Capital, essaye en revanche de penser, avec plus ou moins de succès, la domination en termes de système, recherchant ses dynamiques, ses ruses, ses clivages et ses contradictions internes qui mèneront à la lutte finale. Noble projet, mais qui trahit souvent l'esprit puéril de ces grands stratèges privés d'armée.

8 3. Dans ce contexte, le travail de Luc Boltanski me semble être d'une importance majeure pour comprendre aujourd'hui les défis de la critique. Primo, il est issu de la sociologie de la domination la plus monumentale et la plus puissante de notre époque - celle de Pierre Bourdieu. Comme on le sait, ce dernier essaie de démontrer comment les manières de légitimer les structures de domination sont fondées sur une violence symbolique incarnée dans les réseaux des habitus. La validité de ces structures, qui vont jusqu'à pénétrer le corps même des acteurs, s'avère donc non-négociable et par là même prémunie contre la critique. De virulentes critiques ont été faites à Bourdieu par les défenseurs du pouvoir du peuple comme Jacques Rancière. Le dépassement de la sociologie de la domination proposé par Boltanski est cependant d'un type différent, à la fois empirique et distancié de tout le manichéisme des luttes sociales. Boltanski montre en effet que les structures du pouvoir ne passent pas par des stratégies de justification - elles se nourrissent de la critique, et sont pour la même raison vulnérables à la critique ${ }^{6}$. Secundo, Boltanski - au lieu de proposer sa propre critique stratégique - rend justice à la critique alors en cours dans la dynamique sociale. Par cela, il contribue aussi de manière importante à dégonfler le narcissisme notoire qui hante depuis des siècles la critique sociale des philosophes l'avenir ne dépendant pas tellement de la finesse des théories critiques que des capacités critiques des dominés. Tertio, Luc Boltanski a fait revenir au centre de la réflexion la question du capitalisme comme réalité historique, dynamique et consistante, capitalisme presque invisible aux yeux des micro-critiques dominantes des années 1970-1980. Boltanski a, là aussi, contribué à réhabiliter cette « question capitaliste ${ }^{7}$, qui n'est plus désormais le domaine exclusif des intellectuels d'extrême gauche.

4. Il n'y a pas lieu de choisir entre les différentes grammaires de la critique, tant le pluralisme critique est sans aucun doute enrichissant. Toutefois ce pluralisme ne doit pas et ne peut pas rester pacifique. Dans son livre sur la critique, Luc Boltanski parle d'une contradiction herméneutique de la critique qui est inévitable mais qui n'est pas forcement désarmante. Cette contradiction consiste, pour le dire d'une manière un peu schématique, dans le fait que faire la critique d'une institution (à laquelle on participe d'une manière ou d'une autre) implique la reconnaissance (au moins implicite) de valeurs fondatrices de cette même institution. C'est à la fois la force et la faiblesse de l'entreprise critique. On pourrait pointer ici deux autres paradoxes partageant en quelque sorte cette 
caractéristique. Le premier concerne la fonction stratégique de la critique. Certaines critiques cherchent une meilleure compréhension des systèmes de domination en dénonçant la couche idéologique qui les recouvre. Par là même, ces critiques arrivent parfois à comprendre la domination mieux que celle-ci ne se comprend elle-même. Cette connaissance, censée servir les dominés, devient aussi souvent une arme entre les mains des dominants. Bien des exemples sont connus, parmi lesquels, au rang des plus éminents, figure le Turbocapitalism de Robert Luttwak ou encore l'utilisation des analyses de Deleuze et Guattari par les stratèges de Tsahal'.

Le deuxième paradoxe touche le phénomène du détournement ou tout simplement la récupération. Le potentiel critique se trouve alors absorbé par les structures de domination, les réformant certes, mais les renforçant également. L'histoire de ce que Boltanski appelle la critique artiste ${ }^{9}$ du capitalisme, et le rôle que celle-ci a joué dans la formation d'un nouvel esprit du capitalisme est ici un cas exemplaire.

11 Ces deux paradoxes, inévitables, ne doivent pas nous désarmer. Ils doivent en revanche nous rendre plus modestes en ce qu'ils témoignent du fait qu'il n'y a pas de critique pure. La critique est toujours déchirée entre la volonté du renversement, le rejet pur et muet qui très souvent la motive, et, le geste de l'universalisation. Et cela même au niveau local, qui est formellement ouvert à la réconciliation possible avec ce que fait objet de critique. Il existe encore un troisième pôle - celui de la critique rusée, stratégique ou tactique, qui cherche plus les effet que les justifications. La critique est une entreprise humaine immergée dans l'histoire et vulnérable à ses défauts et ses pièges.

\section{BIBLIOGRAPHIE}

Luc Boltanski, Laurent Thévenot, De la justification. Les économies de la grandeur, Gallimard, Paris, 1991

Luc Boltanski, Eve Chiapello, Le nouvel esprit du capitalisme, Gallimard, Paris, 1999

Luc Boltanski, De la critique, Gallimard, Paris 2009

Pierre Bourdieu, La Distinction ; Critique sociale du jugement, Minuit, Paris, 1979

Michel Foucault, Le courage de la vérité, Hautes Etudes/Gallimard Seuil, Paris 2009

Immanuel Kant, Kritik der reinen Vernunft, Meiner Verlag. Hamburg 1998.

Immanuel Kant, Kritik der praktischen Vernunft. Hrsg. von Horst D. Brandt und Heiner F. Klemme, Meiner, Hamburg 2003

Immanuel Kant, Kritik der Urteilskraft, Meiner Verlag, Hamburg, 1974

Karl Marx, Grundrisse der Kritik der politischen Ökonomie in Marx-Engels-Werkausgabe (MEW) Bd. 42, Seiten 19-875, Dietz, Berlin 1983

Karl Marx, Das Kapital, 1962/1963/1983: in Marx-Engels-Werke (MEW), Band 23-25, Berlin/DDR: Dietz Verlag

Axel Honneth, Kampf um Anerkennung, Suhrkamp Verlag, Frankfurt/M, 1992 
Axel Honneth, Kritik der Macht, Suhrkamp Verlag, Frankfurt/M. 2000

Antonio Negri, Michel Hardt, Empire, Harvard University Press, Harvard, 2000

\section{NOTES}

1. Max Horkheimer, Traditionelle und kritische Theorie, Zeitschift für Socialforschung, Jg.6 (1937)

2. Voir Michel Foucault, Le courage de la vérité. Le gouvernement de soi et des autres II. Hautes Etudes Gallimard Seuil, Paris, 2009, pp. 146-176

3. Luc Boltanski, De la critique, Paris, Gallimard, 2009, pp. 130-134

4. Jurgen Habermas, Strukturwandel der Öffentlichkeit. Untersuchungen zu einer Kategorie der bürgerlichen Gesellschaft Suhrkamp, Neuwied/Berlin 1971 [1962]

5. Axel Honneth, Kampf um Anerkennung: Zur Moralischen Grammatik Sozialer Konflikte. Suhrkamp, Frankfurt am Main 1992

6. Luc Boltanski, Laurent Thévenot, De la justification. Les économies de la grandeur, Paris, Gallimard, 1991

7. Luc Boltankski, Eve Chiapello, Le nouvel esprit du capitalisme, Paris, Gallimard, 1999

8. http://www.frieze.com/issue/article/the_art_of_war/

9. Ibidem pp. 244-249 et 266-280

\section{INDEX}

Mots-clés : critique du travail, correspondance, artistes, socialisme vert, travailleurs culturels, Wertkritik, Gorz André, dialogue, Vincent Jean-Marie, écologie, modèles alternatifs, Marx Karl, penser le Capital, ethos du travail, Eglise Orthodoxe, salariat, domination

\section{AUTEUR}

\section{MICHAL KOZLOWSKI}

Maître de conférences en philosophie contemporaine, Université de Varsovie, journaliste et militant. 